\title{
Automated point clouds processing for deformation monitoring
}

\author{
Ján Erdélyi \\ Slovak University of Technology in Bratislava \\ Department of Surveying \\ jan.erdelyi@stuba.sk
}

\begin{abstract}
The weather conditions and the loading during operation cause changes in the spatial position and in the shape of engineering structures that affect static and dynamic function and reliability of these structures. Because of these facts, geodetic measurements are integral parts of engineering structures' diagnosis.

The advantage of terrestrial laser scanning (TLS) over conventional surveying methods is the efficiency of spatial data acquisition. TLS allows contactless determination of the spatial coordinates of points lying on the surface on the measured object. The scan rate of current scanners (up to 1 million of points per second) allows significant reduction of time, necessary for the measurement; respectively increase the quantity of the information obtained about the object measured. To increase the accuracy of the results, selected parts of the monitored structure can be approximated by single geometric entities using regression. In this case the position of measured point is calculated from tens or hundreds of scanned points.

This paper presents the possibility of deformation monitoring of engineering structures using the technology of TLS. For automated data processing was developed an application based on MATLAB ${ }^{\circledR}$, Displacement TLS. The operation mode, the basic parts of this application and the calculation of displacements are described.
\end{abstract}

Keywords: terrestrial laser scannig, deformation monitoring

\section{Terrestrial Laser Scanning}

The technology of Terrestrial Laser Scanning (TLS) is a non-selective method of spatial data acquisition. TLS determines the 3D coordinates of the measured points on the surface of the measured object in a grid, which is defined by regular angular spacing in the horizontal and vertical directions [8]. The result of TLS is an irregular raster of measured points, the so-called point cloud, which documents the measured object (Fig. 1). The difference between TLS and conventional surveying methods is that the coordinates of characteristic points are obtained by modelling, respectively by generalization of the main elements of 3D models or the resulting point cloud [9].

Most of the current TLS works on the principle of spatial polar method. The spatial position of measured points are calculated from the measured horizontal and vertical angles and from the measured slope distance (Fig. 2 left).

The optimal source of radiation of electromagnetic waves for scanning systems are lasers. These are used for contactless measuring of distances. Laser beams are highly monochromatic

Geoinformatics FCE CTU 14(2), 2015, doi:10.14311/gi.14.2.5 47 


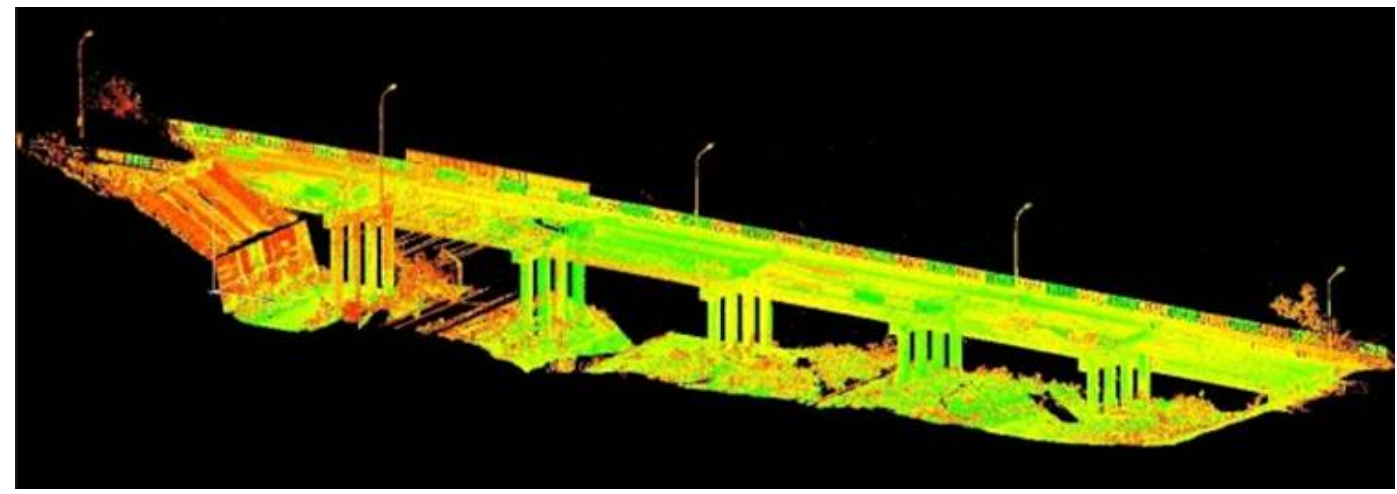

Figure 1: The point cloud of the bridge No. 137, Bojnicka street in Bratislava

and have a narrow spectral line width compared to other sources of radiation.

The deflection of the laser beams is provided by oscillating mirrors, rotating prism, by rotation of the laser source around horizontal and vertical axis of the instrument or by fiber optics, resp. by combination of the methods above mentioned [8]. The most common used is the combination of rotation of instrument around the vertical axis, and an oscillating mirror.
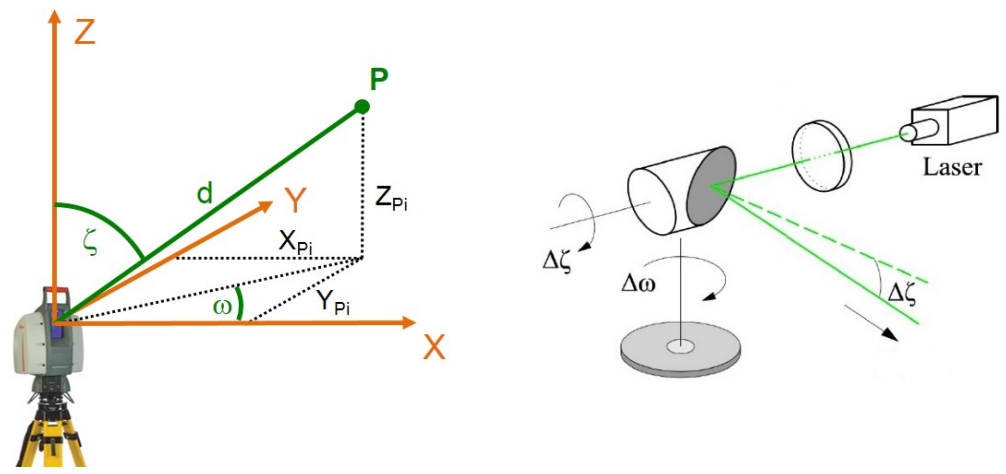

Figure 2: The principle of spatial polar method (left), and the combination of rotating source and oscillating mirror (right)

The process of data acquisition and the modelling using TLS can be divided into three main steps. The first one is the preparation for the measurement (for scanning), recognition of the measured object, the choice of positions for the instrument, signalization of the control points. The second step is the process of scanning and the third one is the processing of data obtained by TLS. The data processing contains:

- Preparation of the point cloud for data processing. This includes initial adjustments of point cloud: error elimination, filtering and data reduction, transformation (between different coordinate systems), elimination of unnecessary points, and coloring of points (assigning the colors according to the intensity of measuring signal or from photographs).

- Processing of data obtained by TLS. Spatial model creation of the measured object or its parts, determination of geometric parameters (e.g. dimensions) and deformations of chosen parts of the measured object. 
- Visualization. Rendering of the created model, and creation of animations.

\section{Deformation monitoring}

The weather conditions and the loading during operation cause changes in the spatial position and in the shape of engineering structures that affect static and dynamic function and reliability of these structures. Because of these facts, geodetic measurements are integral parts of engineering structures' diagnosis $[1,3]$.

One of the ways of deformation monitoring of engineering structures using TLS is the use of differential models $[4,6]$. This method is used to determine the displacements of large surfaces, e.g. surface of the bottom of bridge deck. From the point cloud is created a triangular network at every epoch of measurement. From the differences between them is determined the displacement of the measured structure. These differences are measured in a defined grid in perpendicular direction to the reference plane defined in the epoch of initial measurement [7]. The disadvantage of this method is the lower accuracy, because the triangles are created from the points determined by accuracy of several millimeters (depends on the accuracy of the chosen instrument).

To increase the accuracy of the results of deformation monitoring, selected parts of the monitored structure can be approximated by single geometric entities using regression. In this case the position of the measured points is calculated from tens or hundreds of scanned points. Vertical displacements of the measured points on the bottom part of the selected structures can be determined as the difference between the $\mathrm{Z}$ coordinates of these points in each measurement epoch (Fig. 3). The position of the measured points is modelled by small regression planes using orthogonal regression.

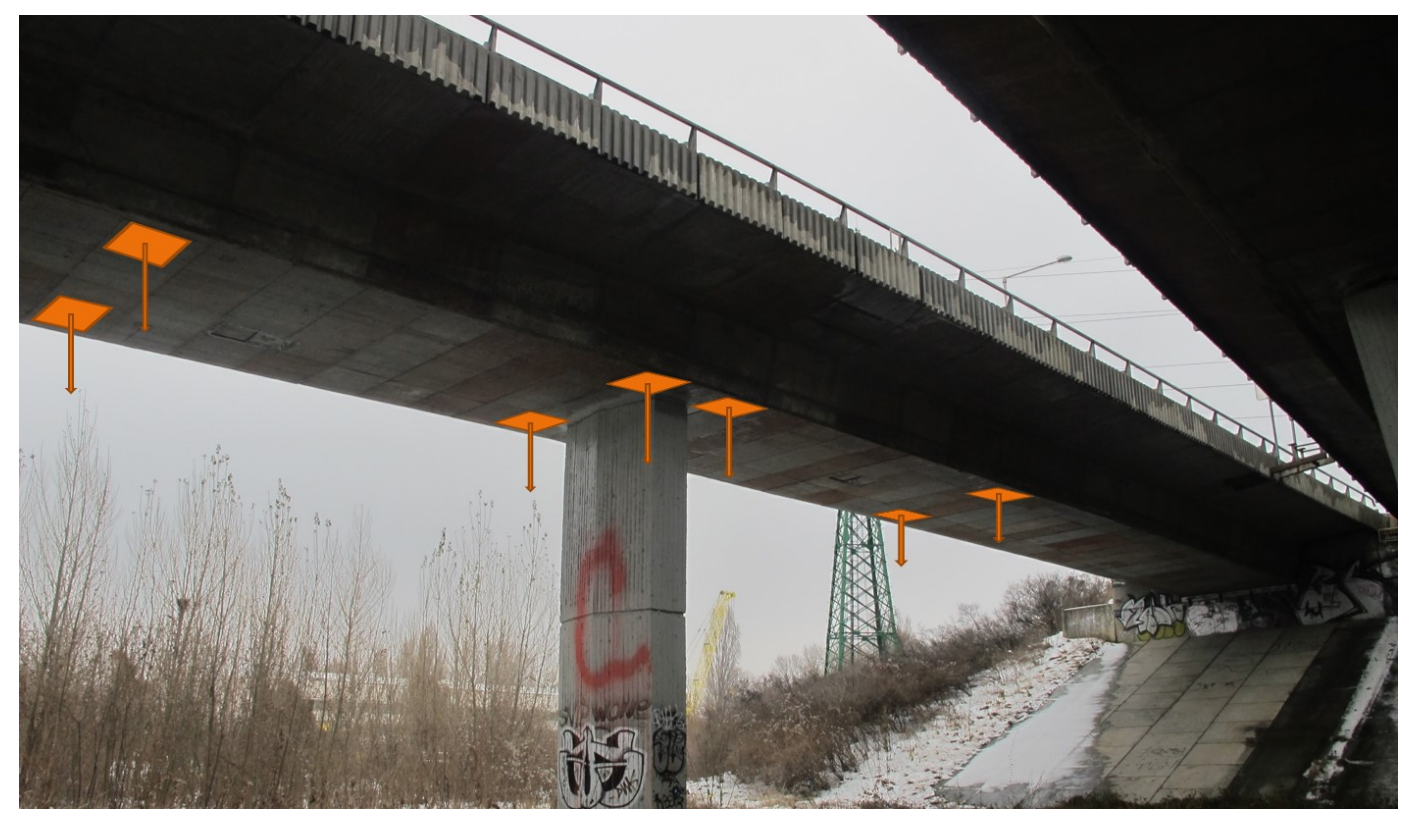

Figure 3: Vertical displacement determination of part of the Harbor Bridge in Bratislava

Orthogonal regression is calculated from the general equation of a plane

Geoinformatics FCE CTU 14(2), 2015 


$$
a \cdot X+b \cdot Y+c \cdot Z+d=0
$$

where $a, b$ and $c$ are the parameters of the normal vector of the plane,

$X, Y$ and $Z$ are the coordinates of the point lying in the plane,

$d$ is the scalar product of the normal vector of the plane and the position vector of any point of the plane.

The orthogonal distance of a point from the plane is calculated by

$$
d_{P, \varrho}=\frac{\left|a \cdot X_{P}+b \cdot Y_{p}+c \cdot Z_{P}+d\right|}{\sqrt{a^{2}+b^{2}+c^{2}}}
$$

The requirement of orthogonal regression is that the sum of the squares of orthogonal distances have to be minimal, so

$$
\sum_{i=1}^{n} \frac{\left|a \cdot X_{i}+b \cdot Y_{i}+c \cdot Z_{i}+d\right|^{2}}{a^{2}+b^{2}+c^{2}}=\min
$$

where $n$ is the number of points used for the calculation of the plane.

Partial derivation of (3) with respect to d leads to

$$
2 \cdot \sum_{i=1}^{n} \frac{\left|a \cdot X_{i}+b \cdot Y_{i}+c \cdot Z_{i}+d\right|}{a^{2}+b^{2}+c^{2}}=0
$$

According to the previous formula, the parameter $\mathrm{d}$ can be formulated as

$$
d=-\left(a \cdot X_{0}+b \cdot Y_{0}+c \cdot Z_{0}\right)
$$

And the formula for the general equation of a plane becomes

$$
a \cdot\left(X_{i}-X_{0}\right)+b \cdot\left(Y_{i}-Y_{0}\right)+c \cdot\left(Z_{i}-Z_{0}\right)=0
$$

where $\left(X_{i}-X_{0}\right),\left(Y_{i}-Y_{0}\right)$ and $\left(Z_{i}-Z_{0}\right)$ are the coordinates of the point cloud reduced to a centroid.

For each point of point cloud is possible to write a formula according to (6), then the design matrix of the system of equations has the form

$$
A=\left(\begin{array}{ccc}
\left(X_{1}-X_{0}\right) & \left(Y_{1}-Y_{0}\right) & \left(Z_{1}-Z_{0}\right) \\
\left(X_{2}-X_{0}\right) & \left(Y_{2}-Y_{0}\right) & \left(Z_{2}-Z_{0}\right) \\
\vdots & \vdots & \vdots \\
\left(X_{n}-X_{0}\right) & \left(Y_{n}-Y_{0}\right) & \left(Z_{n}-Z_{0}\right)
\end{array}\right)
$$

Orthogonal regression is calculated by applying Singular Value Decomposition 


$$
\mathbf{A}=\mathbf{U} \boldsymbol{\Sigma} \mathbf{V}^{T}
$$

where $\mathbf{A}$ is the design matrix, with dimensions $n \times 3$, and $n$ is the number of points used for the calculation. The column vectors of $\mathbf{U}^{n \times n}$ are normalized eigenvectors of matrix $\mathbf{A A}^{T}$. The column vectors of $\mathbf{V}^{3 \times 3}$ are normalized eigenvectors of $\mathbf{A}^{T} \mathbf{A}$. The matrix $\boldsymbol{\Sigma}^{n x 3}$ contains eigenvalues on the diagonals. Then the normal vector of regression plane is the column vector of $\mathbf{V}$ corresponding to the smallest eigenvalue from $\boldsymbol{\Sigma}[2,5]$.

The position of the observed points in XY plane is defined as fixed. The advantage of this procedure is that the position of the measured points does not change with the thermal expansion of the structure. The $\mathrm{Z}$ coordinates (heights) of the measured points are calculated by projecting the points onto regression planes (Fig. 4) using formula

$$
Z_{P}=-\frac{a \cdot X+b \cdot Y+d}{c}
$$

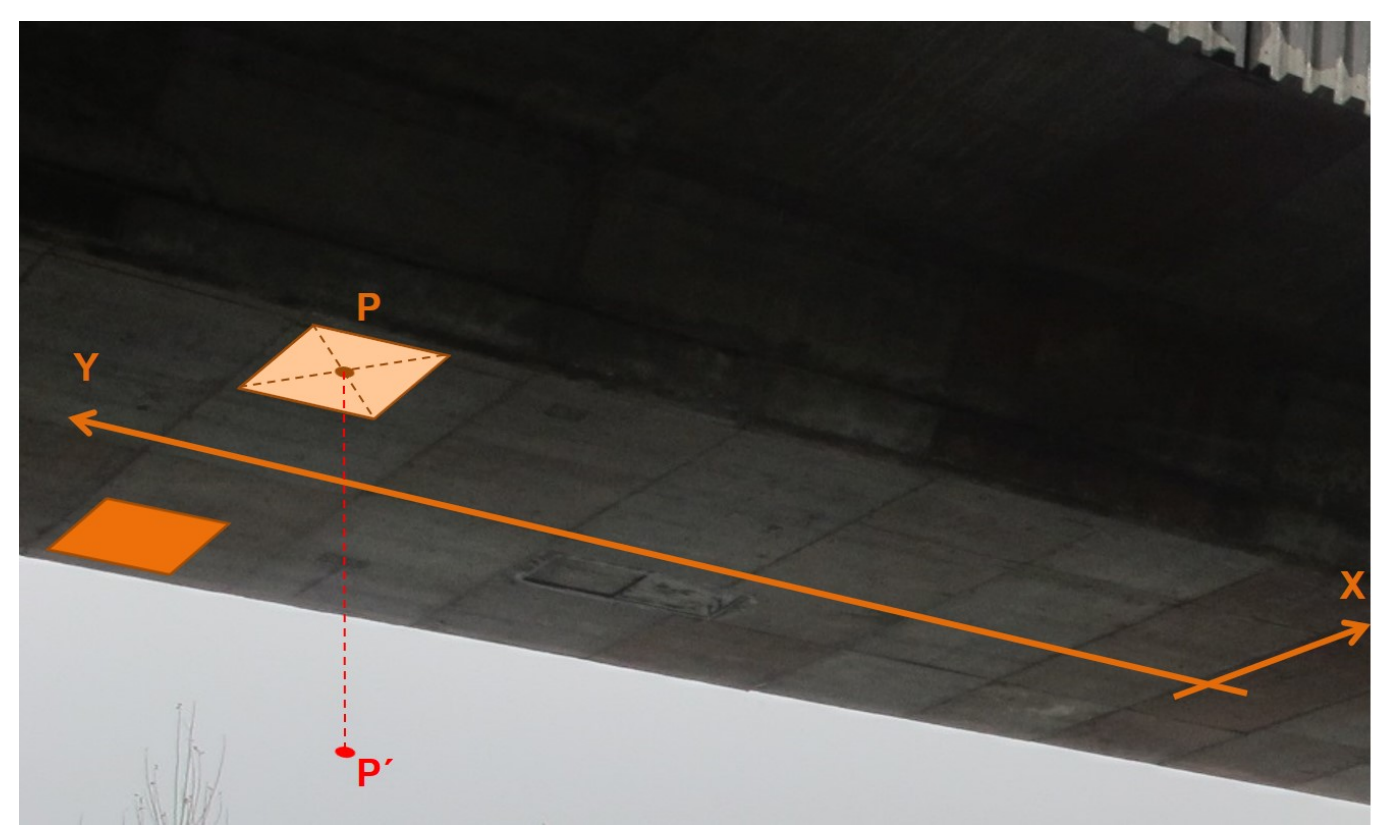

Figure 4: Determination of the height of measured points

The standard deviation of the results are calculated using uncertainty propagation law, from the standard deviation of the vertical component of the transformation error and the standard deviation of the regression planes

$$
\sigma_{Z_{P}}=\sqrt{\sigma_{T_{Z}}^{2}+\sigma_{\varrho}^{2}}
$$

where $\sigma_{T_{Z}}$ is the vertical component of the error of the data transformation and $\sigma_{\varrho}$ is the standard deviation of the calculated regression plane. 
The transformation of the point clouds in each measurement epoch is needed to obtain data in a common coordinate system in each measurement epoch. The accuracy of the transformation is given by the differences $(\Delta \mathrm{X}, \Delta \mathrm{Y}, \Delta \mathrm{Z})$ between the identical reference points after the transformation of the scanned point cloud of the current measurement epoch into the coordinate system of the initial measurement epoch. The standard deviation of the regression planes is calculated from the orthogonal distance of the points of point cloud from these planes. Dispersion of the points around the plane reflects the random error (noise) of the distance measurement by TLS (coordinate determination) mainly. To eliminate the effects of systematic errors it is recommended to perform the measurements during the same conditions in each epoch (position of the scanner, temperature, etc.). The effect of the systematic errors is included in the accuracy of determination of coordinates of the reference points (stable objects in each epoch).

\section{Displacement TLS application}

The proposed procedure of vertical displacement determination, depending on the number of the points of the point cloud, places high demand on computing used for the data processing, as well as on the operator itself (definition of large number of fences, export/import the data, regression calculation, etc.). For the partial automation of the above mentioned procedure, a computational application "Diplacement TLS" was developed (Fig. 5). The application is based on the computational software MATLAB ${ }^{\circledR}$ by MathWorks ${ }^{\circledR}$.

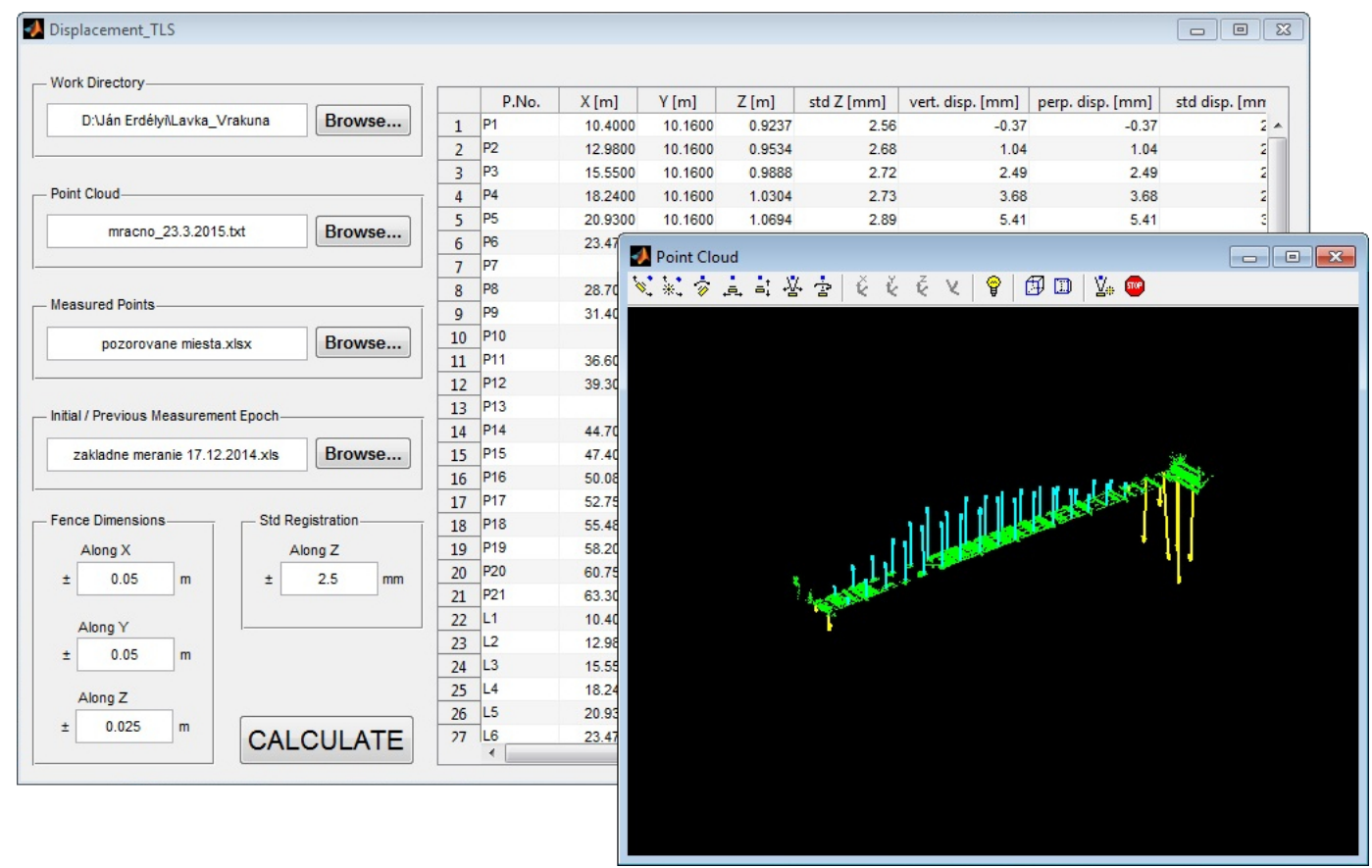

Figure 5: Dialog box of application Displacement TLS

The application was created as a standalone app; however the MATLAB Runtime is necessary to be installed. The work with the app is as follows: In the first step the user can choose a work directory in which the resulting files will be saved. The second step is the point cloud file loading in *.txt or *.xyz file format which contains the coordinates of scanned points. 
The measured points can be arranged in ${ }^{*}$.xls or ${ }^{*}$.xlsx file defining the coordinates of the monitored points. The vertical displacements are calculated in the points defined by the mentioned file and are transformed to perpendicular displacements using the normal vectors of planar surfaces. The user can load the resulting file of the previous measurement epoch in the Initial Previous Measurement box (for comparison of point heights). Without this file the result will be an *.xlsx file containing the heights of the measured points. The fencing boxes, selecting part of the point cloud around the measured points, are defined by its dimensions along axis $\mathrm{X}, \mathrm{Y}$ and $\mathrm{Z}$. These boxes define approximately the same set of points in each measurement epoch. The standard deviation of the registration (vertical component of the error of the data transformation) is necessary for the calculation of the standard deviation of the results using the uncertainty propagation law. The results are shown in the table on the right side of the app's dialog window and are saved into an *.xlsx file in the work directory. The table contains the IDs of the measured points, their coordinates, the standard deviation of the height of measured points ( $\mathrm{Z}$ coordinates), the vertical displacements, the perpendicular displacements and the standard deviation of displacements. A figure which shows the point cloud and the displacement vectors in a relative scale is created for the better imagination of the results.

\subsection{Conclusion}

The issue of inspection of safety operation of engineering structures is always current and closely joined with the activities of surveyors. One of the most important tasks is the determination of the displacements of the selected parts of these structures using different surveying methods. Monitoring of engineering structures can be performed using TLS also. To obtain the accuracy comparable with the accuracy of the conventional surveying methods, selected parts of the monitored structure should be approximated by single geometric entities using regression algorithms.

The paper describes the procedure of displacement determination of engineering structures from point clouds acquired by TLS. The proposed procedure is based on the modelling of the selected part of structures using small planar surfaces by orthogonal regression. The Z coordinates (heights) of the measured points are calculated by projecting the measured points onto regression planes. The vertical displacements of the measured points on the bottom part of the selected structures are determined as the differences between the $\mathrm{Z}$ coordinates of these points in each measurement epoch. This procedure significantly improves the accuracy of the resulting displacements, because the position of the measured point is calculated form tens or hundreds of scanned points, not only from one discrete scanned point.

An application based on software MATLAB ${ }^{\circledR}$ - Displacement TLS was developed for automated data processing. The above mentioned procedure of deformation determination is performed and controlled with help of the graphical user interface of the application. The application was created as a standalone app; however the MATLAB Runtime is necessary to be installed.

\section{Acknowledgement}

This work was supported by the Scientific Grant Agency of the Ministry of Education, science, research and sport of the Slovak Republic and the Slovak Academy of Sciences under the 
contract No.VEGA-1/0445/13.

\section{References}

[1] Jaroslav Braun and Martin Štroner. "Geodetic Measurement of Longitudinal Displacements of the Railway Bridge". In: INGEO 2014 and FIG Regional Central and Eastern European Conference on Engineering Surveying. Vol. 1. ISBN 978-80-01-05469-7. 2014, pp. 231-236.

[2] Aleš Čepek and Jan Pytel. "A Note on Numerical Solutions of Least Squares Adjustment in GNU Project Gama". In: Interfacing Geostatistics and GIS. Springer Science + Business Media, 2009, pp. 173-187. DOI: 10.1007/978-3-540-33236-7_14.

[3] Alojz Kopáčik et al. "Dynamic deformation monitoring of a technological structure". In: Geodetski list 67(90).3 (2013), pp. 161-174. ISSN: 0016-710X.

[4] Bronislav Koska et al. "Monitoring of Lock Chamber Dynamic Deformation." In: Proceeding of Measuring the Changes - 13th FIG Symposium on Deformation Measurements and Analysis and 4th IAG Symposium on Geodesy for Geotechnical and Structural Engineering. LNEC: Lisabon, 2008.

[5] Vladimír Lacko. "Singulárny rozklad matíc a úskalia programovej realizácie Golubovho algoritmu na jeho určenie (Singular Value Decomposition and Difficulties of Software Implementation of Golub Algorithm and its Determination)". In: Student science conference. Comenius University in Bratislava, 2008, p. 69.

[6] Jiří Pospíšil, Bronislav Koska, and Tomáš Křemen. "Using Laser Scanning Technologies for Deformation Measuring". In: Optical 3-D Measurement Techniques. Vol. 2. ISBN 3-906467-67-8. Zurich: Swiss Federal Institute of Technology Zurich, 2007, pp. 226-233.

[7] Thomas Schäfer et al. "Deformation measurement using Terrestrial Laser Scanning at the Hydropower Station of Gabčíkovo". In: INGEO 2004 and FIG Regional Central and Eastern European Conference on Engineering Surveying. [CD-ROM]. Bratislava: KGDE SvF STU, 2004, p. 10. ISBN: 87-90907-34-05.

[8] Martin. Štroner et al. 3D skenovací systémy. ISBN 978-80-01-05371-3. Česká technika - nakladatelství ČVUT, ČVUT v Praze, 2013.

[9] George Vosselman and Hans-Gerd Maas. Airborne and terrestrial laser scanning. Whittles Publishing, 2010, p. 336. ISBN: 978-1904445-87-6. 